\title{
Growing Hyphae of Achlya bisexualis Generate a Longtitudinal pH Gradient in the Surrounding Medium
}

\author{
By NEIL A. R. GOW, 1*+ DARRYL L. KROPF ${ }^{1,2}$ AND \\ FRANKLIN M. HAROLD 1.2 \\ 'Department of Molecular \& Cellular Biology. National Jewish Hospital \& Research Center, \\ Denver, CO 80206, USA \\ ${ }^{2}$ Department of Biochemistry, Biophysics \& Genetics, University of Colorado Health Sciences \\ Center, Denver, CO 80262, U.S.A.
}

(Received 2 May 1984)

Growing hyphae of Achlya bisexualis were found to generate a longitudinal $\mathrm{pH}$ gradient in the surrounding medium; the medium adjacent to the tip was slightly more alkaline than the bulk phase, while that near distal parts was acidic. The profile of external $\mathrm{pH}$ paralleled that of electric current, as measured with a vibrating probe; the apical alkaline zone corresponded to the region of current inflow. In organisms grown in complete medium, both current flow and apical alkalinization were inhibited when amino acid uptake was blocked, either by removing amino acids from the medium or by raising the external $\mathrm{pH}$ to 8.5 . Achlya could, however, adapt to a medium deficient in organic nutrients; elongating hyphae again generated both the pH profile and the transcellular electric current. It is proposed that both the $\mathrm{pH}$ profile and the electric current are manifestations of a transcellular proton current, which arises from the segregation of proton pumps from proton leaks. Symport of protons with amino acids may be one mechanism by which protons enter the hyphal apex.

\section{INTRODUCTION}

Elongation of fungal hyphae is an extreme example of polarized growth, which is well characterized from the ultrastructural viewpoint (Grove \& Bracker, 1970; Howard, 1981; Gooday \& Trinci, 1980; Gooday, 1983). New material reaches the tip in the form of vesicles containing precursors for the production of new cell wall and plasma membrane. The vesicles are produced in the Golgi equivalents and transported to the hyphal apex where they accumulate and fuse with the plasma membrane. The extension zone is rich in microtubules and microfilaments which presumably play a role in the localization of growth. Similar features have been reported for other tip-growing organisms including pollen tubes, certain algae and plants (Sievers \& Schnepf, 1981).

We know little about the physiological gradients that must correspond to the ultrastructural one, with one exception. Many tip-growing organisms generate endogenous electrical currents such that positive charges enter the tip (Jaffe \& Nuccitelli, 1977; Jaffe, 1979, 1981), and this now appears to be true of a variety of fungi as well (Stump et al., 1980; Gow, 1984). In Achlya, which has been quite extensively studied from this point of view (Kropf $e t$ al., 1983, 1984; Armbruster $\&$ Weisenseel, 1983), the transcellular electrical current is thought to be carried by protons, which are pumped out of distal portions of the hypha and leak into the tip.

We report here a phenomenon that appears to be closely related to the transhyphal electrical current. Growing hyphae of Achlya bisexualis generate a longitudinal pH gradient in the external medium, such that the medium surrounding the tip is slightly more alkaline than the bulk phase,

† Present address: Department of Microbiology, University of Aberdeen, Marischal College, Aberdeen AB9 IAS, UK. 
while the distal parts of the hypha are acidic (Kropf et al., 1984). We propose that this pH profile is a second manifestation of the transcellular proton current and arises from the spatial segregation of proton pumps from proton leaks.

\section{METHODS}

Organism and media. Achlya bisexualis female strain T5 was obtained from the American Type Culture Collection. Mycelia were maintained on PYG agar (peptone/yeast extract/glucose) and grown in the defined medium DMA ; the composition of these media is described by Kropf et al. (1984). In some experiments the composition of the DMA medium was modified so that it contained only half the normal concentrations of the constituent amino acids or just a single amino acid at the stated concentration.

Zoospores, used as inocula, were prepared as follows. Four agar discs cut from the margins of growing colonies were placed in $100 \mathrm{ml}$ sporulation medium ( $1 \mathrm{mM} \mathrm{Tris} /$ maleate $\mathrm{pH} 6.5$ containing $1 \mathrm{mM} \mathrm{CaCl}_{2}$ ) and incubated at $29^{\circ} \mathrm{C}$ for $16 \mathrm{~h}$. The concentration of spore preparations was typically 1 to $3 \times 10^{4}$ zoospores $\mathrm{ml}^{-1}$; they could be stored at room temperature for at least $5 \mathrm{~d}$ without significant loss of viability.

Measurement of pH profiles. A I ml sample of spore suspension was diluted into $9 \mathrm{ml}$ of DMA medium, to give about $10^{3}$ zoospores $\mathrm{ml}^{-1}$. Four drops of this suspension were spotted around the margin of a sterile Nucleopore polycarbonate membrane ( $25 \mathrm{~mm}$ diameter, $8 \mu \mathrm{m}$ pore size) which was floating in DMA medium in a Petri dish. The liquid was quickly drawn through the membrane leaving the spores on the upper surface. During overnight incubation at $24^{\circ} \mathrm{C}$ the spores germinated and hyphae grew through the pores of the membrane, vertically down into the growth medium. The membrane was then lifted from the DMA with forceps and washed free of the burfering constituents of DMA by floating the membrane successively in three dishes containing medium of a desired composition.

Measurements of the extracellular pH had to be made in medium of lowered buffering capacity, obtained by omitting both bufter and phosphate from DMA. Care was taken to maintain a constant osmolarity of 26 mosm (that of DMA) throughout an experiment. Removal of osmotically active substances was compensated for by adding mannitol. Floating membranes were then carefully cut with fine scissors into pieces approximately $10 \times 3 \mathrm{~mm}$ and clipped vertically in a specially designed coffin-shaped chamber (volume $5 \mathrm{ml}$ ), so that the hyphae were immersed in test medium and horizontally oriented in the chamber. The chamber was placed on an inverted fixedstage microscope and the hyphae were observed at a magnification of $100 \times$. The $\mathrm{pH}$ profile was mapped with a microelectrode with a pH sensitive tip $50 \mu \mathrm{m}$ in length (M1-102 microelectrode; Microelectrodes, Londonderry, NH, USA). A micromanipulator was used to place the microelectrode gently against the surface of a hypha at different positions and the local $\mathrm{pH}$ was compared to that of reference positions in the medium at least $300 \mu \mathrm{m}$ from the hypha. We observed no artefacts attributable to the practice of touching the hypha with the electrode. The output from a Radiometer PHM 26 meter was recorded in $\mathrm{mV}$ and the readings were calibrated using media of standardized $\mathrm{pH}$.

To exchange the medium during an experiment, new medium was dripped in at one end of the chamber and aspirated from the other. Efficient exchange was facilitated by the shape of the chamber. Preliminary experiments, in which removal of potassium ions was monitored using a cation sensitive electrode, showed that $110 \mathrm{ml}$ of medium had to be passed through the chamber to reduce the potassium concentration by a factor of at least $10^{4}$. This was the minimum volume used whenever media were exchanged.

Measurement of the transhyphal electrical current. Hyphae were grown through polycarbonate membranes and prepared for examination as above. The extracellular current flow was mapped with a vibrating probe (Jaffe \& Nuccitelli, 1974). The instrument and its application to Achlya have been described by Kropf et al. (1984).

\section{RESULTS}

\section{pH profiles along growing hyphae}

A hypha with a pH microelectrode in recording position is shown in Fig. I (a), and a set of measurements recorded along a growing hypha in Fig. $1(b)$. The pH immediately external to the apex of a growing hypha was always alkaline with respect to the bulk medium, but acidic distally. Hyphae that were not elongating lacked the alkaline zone around the tip (see below).

Fig. 2 compares the longitudinal profiles of $\mathrm{pH}(a)$ and electrical current $(b)$ recorded from a single growing hypha. The zone of alkalinity around the tip matched that of inward current, but the distal acidic zone extended farther than the region of outward current. Pooled results from a number of experiments performed under the conditions of Figs 1 and 2 indicate that the average length of the alkaline apical region ( $\mu \mathrm{m} \pm \mathrm{SD}$ ) was $180 \pm 130$. Hyphae were acidic as far behind this region as could be measured (furthest point, $2000 \mu \mathrm{m}$ ). The average position of maximum 


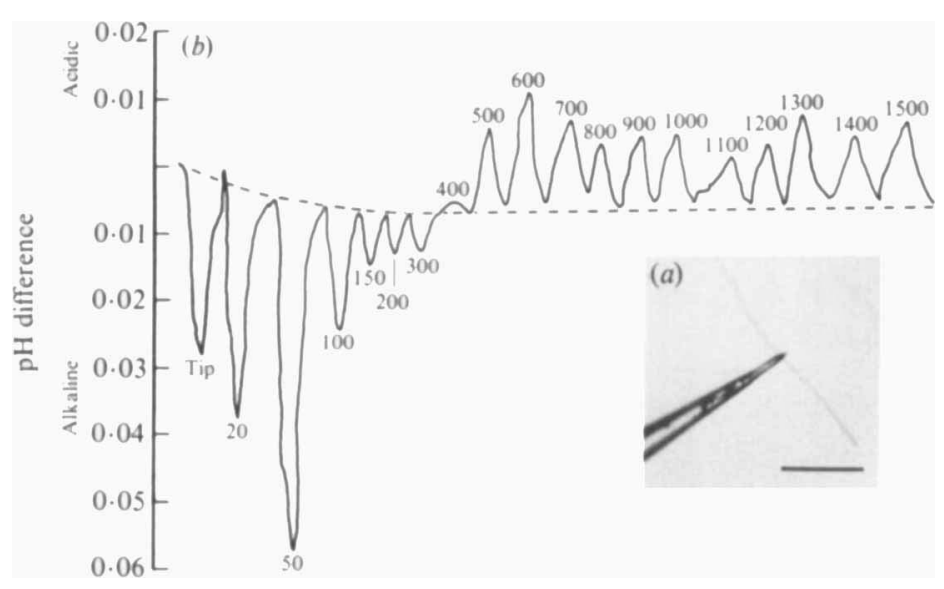

Fig. 1. Measurement of the pH profile along a hypha of A. bisexualis. (a) Photomicrograph of a $\mathrm{pH}$ microelectrode in recording positions on a hypha. Bar marker, $200 \mu \mathrm{m}$. (b) Chart recording of a series of pH defiections, measured as a microelectrode was moved successively between positions adjacent to the hypha and reference positions in the external medium (at the broken line). The numbers on the peaks are the distances of the electrode behind the hyphal apex, in $\mu \mathrm{m}$. The hypha was growing in unbuffered, phosphate-deficient DMA, pH 6.5, with half-normal concentrations of the amino acids.

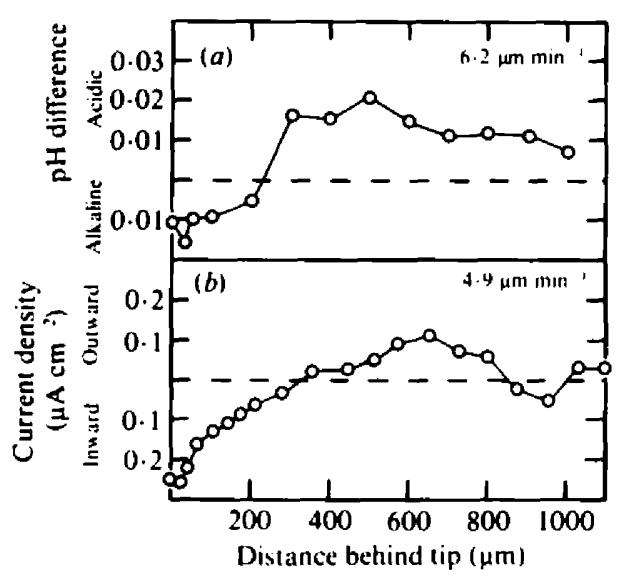

Fig. 2. Comparison of the pH (a) and the electrical current $(b)$ profiles of the same hypha. The growth medium contained unbufiered, phosphate-deficient DMA (pH 6.5), with half-normal concentrations of the amino acids. The numbers in the top right of the panels are the growth rates of the hypha, measured while recording the profiles. Both profiles were recorded within a period of $45 \mathrm{~min}$.

alkalinization occurred $38 \mu \mathrm{m}$ behind the apex, in good agreement with the peak of inward current, 30 to $60 \mu \mathrm{m}$ behind the apex (Kropf et al., 1984). The average values for the peaks of alkalinity and acidity were 0.022 and $0.028 \mathrm{pH}$ units, respectively (largest deflections were 0.086 and $0.095 \mathrm{pH}$ units, respectively). Those hyphae with larger than average alkaline deflections (e.g. Fig. $1 b$ ) tended to have extended alkaline regions.

\section{Dependence of $\mathrm{pH}$ profiles on amino acids and external $\mathrm{pH}$}

In an earlier study with $A$. bisexualis, Kropf et al. (1983) showed that, in organisms grown on complete medium, the transhyphal electrical current required the presence of amino acids and was abolished by raising the external pH from 6.5 to 8.5. Fig. 3 documents parallel experiments on the generation of the extracellular $\mathrm{pH}$ profile. 

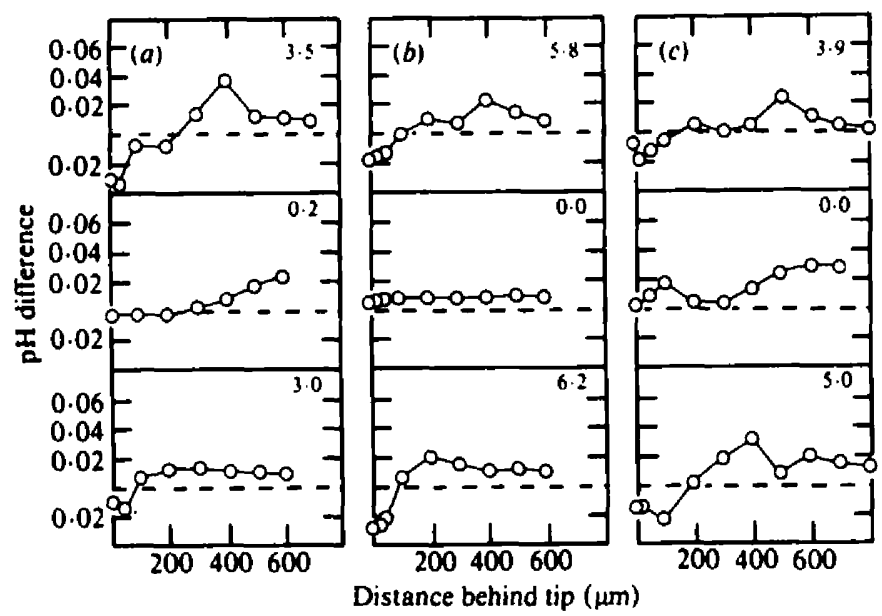

Fig. 3. Effect of amino acids and external pH on the extracellular pH profiles of hyphae. Deflections below and above the broken line indicate alkalinity and acidity, respectively. The profiles of $\mathrm{pH}$ were determined as described in Methods. The elongation rates of the hyphac, in $\mu \mathrm{m} \mathrm{min}^{-1}$, are shown in the top right of each panel. (a) Amino acids. Hyphae grown in DMA were transferred to medium lacking phosphate and buffer but containing half the normal concentration of amino acids. The profile at the top was determined in the presence of these amino acids, that in the middle after all amino acids had been removed, and the profile at the bottom when the amino acids had been restored. In all cases the pH of the medium was 6.5. (b) External pH. Hyphae were grown as above, but instead of removing amino acids, the external $\mathrm{pH}$ was changed. The upper profile was determined at pH 6.5, the middle one at pH 8.5, and the bottom profile after restoration to pH 6.5. (c) Methionine. Hyphae were grown on DMA overnight and then incubated for about $2 \mathrm{~h}$ in modified DMA lacking bufier, phosphate and amino acids, but supplemented with 1 mu-methionine; the pH was 6.5. The upper profile was measured in the presence of methionine, the middle profile after removal of methionine, and the bottom profile after methionine had been restored.

When the complete medium was replaced by one lacking the amino acids, the hyphae ceased to elongate and the alkaline zone around the apices was no longer detectable; distal acidity, however, persisted. As soon as the amino acids were restored, the hyphae resumed growth and recovered an alkaline zone (Fig. $3 a$ ). The changes in both elongation rate and $\mathrm{pH}$ profile occurred within the 10 min required to exchange the medium in the chamber. By contrast to the drastic effects of amino acid removal, removal of glucose affected neither elongation nor the $\mathrm{pH}$ profile (data not shown).

The effect of the pH of the medium on the longitudinal pH profile is illustrated in Fig. 3(b). When the normal medium, pH 6.5, was replaced by the same medium at $\mathrm{pH} 8.5$, the hyphae stopped growing and ceased to generate an alkaline zone around the tip, but the distal region remained acidic. Both elongation and apical alkalinization resumed as soon as the original medium at pH 6.5 was re-introduced into the chamber.

Equivalent results were obtained with organisms growing on modified DMA medium containing only one amino acid. Hyphae were grown on DMA overnight, and then incubated for $2 \mathrm{~h}$ in a modified medium at $\mathrm{pH} 6.5$ lacking buffer, phosphate and amino acids but supplemented with $1 \mathrm{mM}$-methionine. When the methionine was removed, elongation ceased and the alkaline zone around the tip was lost; both elongation and alkalinity recovered when methionine was restored (Fig. 3c). Three methionine analogues (methionine sulphoxide, methionine sulphoximine and D-methionine) supported neither current flow nor apical alkalinity (data not shown).

Fig. 3 shows observations made with individual hyphae, but the correlation between the presence of amino acids, hyphal extension and alkalinity around the tip is supported by the accumulated data from several experiments. Out of 120 hyphae examined in the presence of one or more amino acids, $95 \%$ had alkaline tips and the average extension rate was $3.7 \mu \mathrm{m} \mathrm{min}^{-1}$. The few 


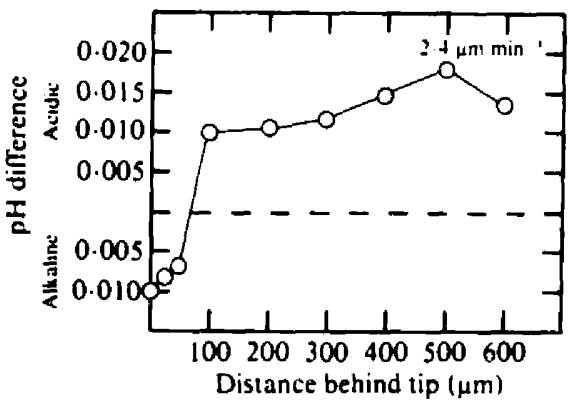

Fig. 4

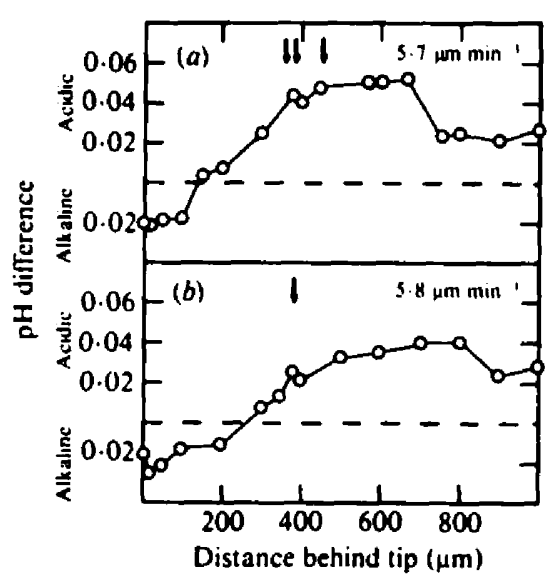

Fig. 5

Fig. 4. Extracellular pH profile and growth rate of a hypha growing in the absence of added nutrients. Hyphae were first grown in DMA then transferred to media containing salts but no phosphate, buffer glucose or amino acids for several hours until growth had resumed.

Fig. 5. Effect of branching on the pH profile of a hypha. For growth conditions see text. Branches formed at positions indicated by the arrows. Profile (b) was measured 62 min after $(a)$; all branches were less than $10 \mu \mathrm{m}$ long at the time the $\mathrm{pH}$ profiles were mapped.

hyphae whose tips were neutral or even acidic grew slowly, or not at all. Out of 24 profiles measured immediately after the removal of amino acids, only 2 hyphae had tips that were slightly alkaline; 22 had acidic or neutral tips, and their average extension rate was only $0.8 \mu \mathrm{m} \mathrm{min}^{-1}$.

\section{Adaptation to medium lacking amino acids}

In the experiments described above, Achlya was grown on medium containing one or more amino acids; when the amino acid(s) was withdrawn, elongation stopped and the cells ceased to generate the alkaline apical zone. The transhyphal electrical current was also abolished by removal of amino acids (Kropf $e t$ al., 1984). However, after about an hour in the absence of all organic nutrients, elongation resumed, as did the generation of apical alkalinity (Fig. 4) and electrical current. Even under these conditions, apical extension and alkalinity ceased when the external $\mathrm{pH}$ was raised from 6.5 to 8.5 (data not shown). The salient point is that, regardless of the composition of the medium, elongating hyphae generate both apical alkalinity and a transcellular electrical current.

\section{Effect of branching on the $\mathrm{pH}$ profile}

In the growth media used here, a new zone of inward electrical current precedes the emergence of a branch and predicts its location. When a branch forms, the intensity of inward current at the parent hyphal tip diminishes and may even reverse; the original tip continues to grow even if the electrical current turns outward (Kropf et al., 1983). Mapping of the pH profile is less sensitive than that of the electrical current, and yields only semi-quantitative data. However, within the limits of our methodology it appears that, unlike the electrical current pattern, the pH profile was not altered during branching.

Branching occurred infrequently in media containing a single amino acid and no phosphate, making studies on branching difficult. The frequency of branching was, however, stimulated threefold in media with half-normal concentrations of all acids in DMA plus $20 \mu \mathrm{M}$-phosphate. This small amount of phosphate buffer did not interfere with mapping of the $\mathrm{pH}$ profiles. Branches formed most frequently 300 to $400 \mu \mathrm{m}$ from the hyphal apex but were sometimes produced more than a millimeter from the tip. Branches frequently formed in a group; apical branches were never observed. 
Formation of a branch had no effect on the $\mathrm{pH}$ profile at the parent hyphal tip (Fig. 5); in 45 branching events, the $\mathrm{pH}$ at the parent hyphal apex remained alkaline. Since an alkaline zone always surrounds an elongating tip, one may also expect a localized region of alkalinity at the locus of branching. We found, however, that branches always formed within the acidic regions of hyphae and produced neither detectable alkalinization not a saddle in the acidity at that point.

\section{DISCUSSION}

Growing hyphae of $A$. bisexualis generate a longitudinal pH gradient in the external medium, such that the zone surrounding the apex is slightly more alkaline than the bulk medium, while more distal regions are slightly acidic. This pH profile is the result of at least two dissociable processes. Hyphae produce acid in both the presence and absence of organic nutrients, and whether they are elongating or not. We believe that this acid production results from the efflux of acidic metabolites, such as lactic or succinic acids, and will not consider it further. Our concern here is with the genesis of the alkaline zone around the tip, which is closely linked to hyphal elongation. Various treatments that halted elongation also abolished the apical alkalinity; when elongation resumed, apical alkalinity reappeared. In principle, several physiological processes could give rise to the longitudinal $\mathrm{pH}$ profile, and their identification is hampered by the close coupling of the $\mathrm{pH}$ profile to elongation.

It is likely, for example, that metabolism is longitudinally differentiated. Zalokar (1958) reported early observations along this line; it is now well known that chitin synthase is activated locally and is dormant elsewhere (Gooday, 1978, 1983; Cabib et al., 1979). Similarly, it could be speculated that hyphae release ammonia at the tip by the oxidation of exogenous amino acids or by the breakdown of endogenous proteins. This possibility cannot be excluded, but it fails to explain the correlation between apical alkalinity, growth and the transcellular electrical current.

A more intriguing hypothesis stems directly from the nature of apical growth, which involves the transport of numerous cytoplasmic vesicles to the tip where they fuse with the plasma membrane and externalize their contents. If the vesicular contents have the same $\mathrm{pH}$ as the cytoplasm, around pH 7.5 (Smith \& Raven, 1979; Sanders \& Slayman, 1982), elongation itself will contribute to apical alkalinity. The magnitude of this contribution can be estimated to a first approximation, as follows. Apical vesicles in Achlya range from 80 to $150 \mathrm{~nm}$ in diameter (Hill \& Mullins, 1980); we assume an average diameter of $100 \mathrm{~nm}$. In order to account for the area of plasmalemma that is added when hyphae grow at $5 \mu \mathrm{m} \mathrm{min}^{-1}, 5000$ such vesicles must fuse with the tip per min. Suppose, furthermore, that the vesicles contain $0.1 \mathrm{M}$-buffer, $\mathrm{pK}_{\mathrm{a}}=8$, at $\mathrm{pH} 8$ (a generous assumption). One can readily calculate that each hypha will externalize $2.5 \times$ $10^{-4} \mathrm{pmol}$ buffer $\mathrm{min}^{-1}$, which would neutralize $1.25 \times 10^{-4} \mathrm{pmol} \mathrm{H}^{+}$at the surface. This number must be compared with the number of protons that will diffuse into the apical alkaline zone from the surrounding medium, a flux estimated at $60 \times 10^{-4} \mathrm{pmol} \mathrm{min}^{-1}$ by Kropf et al. (1984). To put it another way, only if the vesicles contained at least $1 \mathrm{M}$-alkaline buffer could they plausibly account for the maintenance of apical alkalinity. In fact, the $\mathrm{pH}$ of apical vesicles is unknown; cytochemical observations suggest that the apical cytoplasm is acidic (Turian, 1979, 1981).

We therefore favour a third hypothesis, which attributes the generation of an alkaline zone around the hyphal tip to the net uptake of protons by the apical region. In an earlier study, Kropf et al. (1984) inferred from measurements with the vibrating probe that Achlya derives a current of protons through itself, such that protons enter the tip and are expelled distally. In DMA medium, proton uptake by the tip required the presence of amino acids; raising the $\mathrm{pH}$ from 6.5 to 8.5 abolished both the proton current and the uptake of amino acids. Kropf et al. (1984) proposed that protons are expelled by a proton-translocating ATPase and flow back into the tip by symport with amino acids. According to this hypothesis, the hypha is a spatially extended chemiosmotic system, with the symporters preferentially located in the region of the tip. This premise led us to predict, and to search for, apical alkalinity. The extent of alkalinization, about $0.02 \mathrm{pH}$ units, is quantitatively consistent with an influx of protons at a rate given by the inward electrical current of 0.5 to $2.5 \mu \mathrm{A} \mathrm{cm}^{-2}$ (Kropf et al., 1984). 


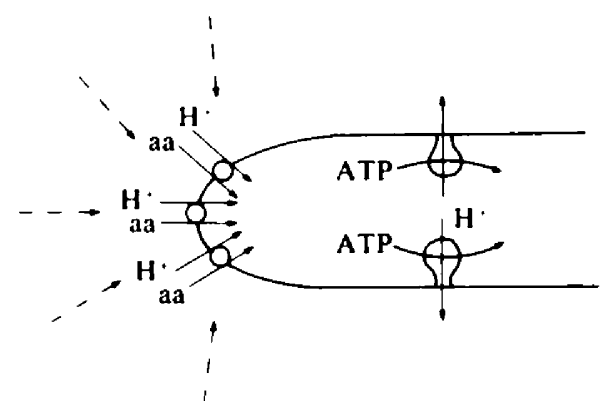

Fig. 6. Proposed model for establishment of the measured extracellular profiles of $\mathrm{pH}$ and currents around a hypha. Protons enter the hyphal tip by amino acid symport and are expelled distally by proton pumping ATPases. Localized symport causes depletion of protons around tip and protons diffuse slowly down the induced concentration gradient (arrows). The apex is therefore relatively alkaline, and the distal region acidic. The net flow of positive charges is into the apex and out from the rear.

The hypothesis that apical alkalinity is a direct manifestation of the transcellular proton current is supported by the close correspondence between the $\mathrm{pH}$ profile and the flow of electrical current. (i) The extent of the alkaline zone was in good agreement with that of inward current (Fig. 2). (ii) When hyphae were grown in DMA medium, both inward current and apical alkalinity were abolished by withdrawal of the amino acids or by raising the $\mathrm{pH}$ of the medium (Fig. 3). The same was true when L-methionine was the sole amino acid present. In contrast to the findings with amino acids, omission of glucose diminished neither the electrical current nor apical alkalinity. (iii) Both apical alkalinity and the transhyphal electrical current were abolished concurrently by addition of cycloheximide (data not shown). (iv) After a period of 1 to $2 \mathrm{~h}$, Achlya hyphae adapt to nutritionally deficient media; elongation resumes and both apical alkalinity and the electrical current flow were observed (data not shown; see also Armbruster \& Weisenseel, 1983). We conclude that both apical alkalinity and the inward current result from the entry of protons into growing hyphal tips.

The mechanism by which protons traverse the plasma membrane is a separate issue, which has not been fully resolved. Most of the data are consistent with the proposal (Kropf et al., 1984) that protons enter by symport with amino acids (Fig. 6), a mechanism well attested in fungi (Eddy, 1982; Harold, 1982; Goffeau \& Slayman, 1981). Hyphae can, however, adapt to media lacking amino acids or other organic nutrients; they elongate for some time and generate both apical alkalinity and a transcellular electrical current. We believe that these phenomena indicate an alternative mode of proton entry into the tip, whose nature is presently unknown. Existence of such a transport pathway would not be surprising, since endogenous energy reserves could support the continued generation of a proton current by the proton-translocating ATPase.

If apical alkalinity results from the uptake of protons, it can be used as a qualitative indicator of the proton flux. As such, it may sometimes detect proton influx that is missed by the vibrating probe. For instance, when Achlya hyphae branch, the fiow of electrical current into the original tip diminishes and may even reverse (Kropf et al., 1983). However, as reported here, growing tips generate apical alkalinity whether they are branching or not, suggesting that protons always flow into a growing tip. Reversal of the electrical current during branching may be due to other ion fluxes that mask the inward current carried by protons (Kropf et al., 1983), but the ions in question remain to be identified. Similarly, from the finding that a new point of current entry precedes and predicts the emergence of a branch (Kropf et al., 1983), one would expect a zone of alkalinity to appear at the same locus. Attempts to corroborate this expectation were unsuccessful, perhaps because metabolic acid swamps the small proton influx.

The present findings leave unresolved the question whether proton influx, aside from its thermodynamic role in driving nutrient uptake (Mitchell, 1976; Harold, 1982; Eddy, 1982) also plays a part in localizing or maintaining tip growth. But we would emphasize that, under all the conditions tried so far, it appears that when a tip grows, protons flow into it. 
We gratefully acknowledge useful discussions with John $\mathbf{H}$. Caldwell and Ruth Harold. We thank W. J. Betz for computer assistance and R. Kennedy for fabricating the coffin-chamber. This research was supported by a Public Health Service grant AI-03568 from the National Institute of Allergy and Infectious Diseases, and a National Science Foundation grant PCM 8009439 from the Metabolic Biology Programme.

\section{REFERENCES}

ARuanuster, B. L. \& Welsensegl, M. H. (1983). Ionic currents traverse growing hyphae and sporangia of the mycelial water mold Achyla debaryana. Protoplasma 115, 65-69.

Cabib, E., Duran, A. \& Bowers, B. (1979). Localized activation of chitin synthetase in the initiation of yeast septum formation. In Fungal Walls and Hyphal Growh, pp. 189-201. Edited by J. H. Burnett, \& A. P. J. Trinci. Cambridge: Cambridge University Press.

EDDY, A. A. (1982). Mechanisms of solute transport in microorganisms. Advances in Microbial Physiology 23, 1-78.

Goppiau, A. Slayman, C. W. (1981). The protontranslocating ATPase of the fungal plasma membrane. Biochimica et biophysica acta 639, 197223.

Gooday, G. W. (1978). The enzymology of hyphal growth. In The Filamentous Fungi, vol. 3, pp. 51-77. Edited by J. E. Smith \& D. R. Berry. London: Edward Amold.

Gooday, G. W. (1983). The hyphal tip. In Recent Advances in Fungal Morphogenesis, pp. 315-356. Edited by J. E. Smith. New York: Marcel Dekker.

GoOdAy, G. W. TRinci, A. P. J. (1980). Wall structure and biosynthesis in fungi. Symposia of the Society for General Microbiology 30, 207-251.

Gow, N. A. R. (1984). Transhyphal electrical currents in fungi. Joumal of General Microbiology (in the Press).

Grove, S. N. \& Bracker, C. E. (1980). Protoplasmic organization of hyphal tips among fungi; vesicles and Spitzenkörper. Journal of Bacteriology 104, 9891009.

HaRold, F. M. (1982). Pumps and currents; a biological perspective. Current Topics in Membranes and Transport 16, 485-516.

Hill, T. W. \& Mulliss, J. T. (1980). Hyphal tip growth in Achlya. I. Cytoplasmic organization. Canadian Journal of Microbiology 26, 1132-1140.

HOWARD, R. J. (1981). Ultrastructural analysis of hyphal tip cell growth in fungi; Spitzenkorper. Journal of Cell Science 48, 89-103.

JAFFE, L. F. (1979). Control of development by ionic currents. In Membrane Transduction Mechanisms. Society for General Physiologists Series, vol. 33, pp. 199-231. Edited by R. A. Cone \& J. E. Dowling, New York: Raven Press.
JAFFE, L. F. (1981). The role of ionic currents in establishing developmental pattern. Philosophical Transactions of the Royal Society of London B29s, 553-566.

JAFFE, L. F. \& Nuccitelli, R. (1974). An ultrasensitive vibrating probe for measuring steady extracellular currents. Journal of Cell Biology 63, 614-628.

JAFPE, L. F. \& Nuccrtelli, R. (1977). Electrical controls of development. Anmual Review of Biophysics and Bioengineering 6, 445-476.

Kropf, D. L., Lupa, M. D. A., Caldwell, J. H. \& Harold, F. M. (1983). Cell polarity: Endogenous ion currents precede and predict branching in the water mold Achlya. Science 220, 1385-1387.

Kropf, D. L., Caldwell, J. H., Gow, N. A. R. \& HAROLD, F. M. (1984). Symport of protons with amino acids carries transcellular current into hyphal tips. Journal of Cell Biology 99, 486-496.

Mitchell, P. (1976). Vectorial chemistry and molecular mechanics of chemiosmotic coupling: power transmission by proticity. Biochemical Society Transactions 4, 399-439.

Sanders, D. L. \& Slayman, C. L. (1982). Control of intracellular pH. Predominant role of oxidative metabolism, not proton transport, in the eukaryotic microorganism Neurospora. Journal of General Physiology 80, 377-402.

Sievers, A. \& SCHNEPF, E. (1981). Morphogenesis and polarity of tubular cells with tip growth. In Cytomorphogenesis in Plants, 8, pp. 265-299. Edited by $O$. Kiermager Vienna: Springer Verlag.

SMTt, F. A. \& RAveN, J. A. (1979). Intracellular pH and its regulation. Annual Review of Plant Physiology 30, 289-311.

StUmp, R. F., Robinson, K. R., Harold, R. L. \& HAROLD, F. M. (1980). Endogenous electrical currents in the water mold Blastocladiella emersonii during growth and sporulation. Proceedings of the National Academy of Sciences of the United States of America 77, 6673-6677.

TuRLN, G. (1979). Cytochemical gradients and mitochondrial exclusion in the apices of vegetative hyphae. Experientia 35, 1164-1166.

TURLAN, G. (1981). Low pH in fungal bud initials. Experientia 37, 1278-1279.

ZALOKAR, M. (1959). Enzyme activity and cell differentiation in Neurospora. American Journal of Botany 46, 555-559. 